\title{
Depth Control of Autonomous Underwater Vehicle Using Discrete Time Sliding Mode Controller
}

\author{
Nira Mawangi Sarif, Rafidah Ngadengon", Herdawatie Abdul Kadir, Mohd Hafiz A.Jalil \\ Faculty of Electrical Engineering, Universiti Tun Hussein Onn Malaysia, Malaysia
}

Received July 25, 2019; Revised October 3, 2019; Accepted December 10, 2019

Copyright $\odot 2019$ by authors, all rights reserved. Authors agree that this article remains permanently open access under the terms of the Creative Commons Attribution License 4.0 International License

\begin{abstract}
This study presents a Discrete Time Sliding Mode Controller (DSMC) application on depth plane of Autonomous Underwater Vehicle (AUV). The main contribution on this work is an implementation of DSMC on NSP AUV II. Sliding Mode Control (SMC) is a robust type of controller and certainly suitable for controlling AUV in the presence of environmental disturbances and uncertainties. DSMC preserves the properties of standard SMC. Linearized dynamic model of NSP AUV II is used in the numerical simulations. Discrete Proportional Integral Derivative (PID) controllers are used for performance comparative analysis. The design of discrete PID and DSMC for NSP AUV II depth is described. Comparative study between the control laws is presented. The simulated results illustrate strong robustness, improve performance and satisfactory stability of DSMC as compared to discrete-time PID controller.
\end{abstract}

Keywords Autonomous Underwater Vehicle, Depth Control, Discrete Sliding Mode Controller, Discrete Proportional-Integral-Derivative

\section{Introduction}

Autonomous Underwater Vehicle (AUV) has shown popularity for three decades due to its versatility and excellent performance which is increasingly being used in many industries [1]. Their solid small size with self-operated propulsion systems, capability carrying sensors such as depth sensors, video cameras, side-scan sonar and other oceanographic measuring devices makes AUV be well suited in dangerous mission. Futuristic element in AUVs prompts advantage to wider area such as surveillance, environmental monitoring, underwater inspection of harbor and pipeline, geological and biological survey, mine counter measures and so forth. However, an extremely unexpected ocean behavior created challenges to
AUV navigation and motion performance in which this phenomenon demonstrates highly frequency oscillating movement that not only affects the sensor performance especially acoustical and optical sensors but also creates the dynamics system into highly nonlinear, time-varying and uncertainties in hydrodynamic parameters such as added mass, lift forces, gravity and buoyancy forces [2]. Additionally, most AUVs are operated on under actuated mode, hence tracking and stabilization control become demanding task, owing to over possession degree of freedom (DOF) beyond control [3]. Furthermore, this limitation is imposed in real life application due to inverting or pointing vertically that can cause equipment damage or dangerous control response [4]. As a result, AUVs motion control is restricted to only one noninteracting subsystem at a time [5]. Due to aforementioned challenges, many advanced control techniques have been implemented in existing literatures, mostly including robust control techniques in [6]-[8] intelligent control method in [9] and adaptive control approach in [10]-[12]. It's apparent that among robust controller types, SMC evidently considers a promising strategy [13] to overcome above obstacles.

The work reported in the literature addresses, the majority of the SMC application on AUV is in continuous time point of view but their effectiveness in real situations cannot be guaranteed [13]. This is because, all of these systems are operated on the discrete time domain in practice with utilization on digital computers or microprocessors, hence continuous time control cannot be implemented [13]. As a result, discrete time sliding mode control (DSMC) has produced significant interest over recent years [13]-[15] in solving the problems caused by the discretization of continuous time controllers. Started in 1997, Lee [16] adopted self-tuning discrete sliding mode control on AUV ARMA based on equivalent discrete variable structure control method and continue research on quasi sliding mode control in presence of uncertainties and long sampling interval in [17] on AUV VORAM. Followed 
by Shaowei Zhang [18] proposed discrete-time quasi sliding mode controller for the multiple-input multiple-output on AUV REMUS. Lastly, Baoju [19] implemented adaptive sliding mode control in discrete time system and applied time varying sliding surface obtained by parameter estimation method.

Research in discrete-time controller designed has started by Dote and Hoft [20] that introduced reaching condition. Later Sarpturk, et al [21] revised Dote and Hoft reaching condition and founded equivalent form based on Lyapunov method. Then, Furuta [22] proposed another reaching condition on the basis of Lyapunov function. Finally, quasi sliding mode band was created by Gao [23] based on non-switching reaching condition and Bartoszewics [24] based on non-switching condition. Although Gao's reaching law method has been introduced since two decades ago, it's still been used in many significant studies such as [25]-[27].

The main aim on this research is to implement reaching law proposed in [26] on depth motion control. Discrete PID and DSMC using reaching law by Gao's in [28] are tested on AUV NSP II via simulation. Discrete PID controller is used for performance comparative analysis. The paper is organized as follow: Section 2 introduced dynamic model of AUV NSP II in the Body-Fixed Reference frame (BFF). Section 3 presents discrete sliding mode control structure designed. Results from numerical simulation are illustrated in section 4 and section 5 providing discussion on advantages of the control methods.

\section{Mathematical Model of AUV NPS II}

\subsection{Nonlinear General Equation of Motion}

According to Fossen [29], general motion of AUV can be described by using BFF and earth-fixed frame (EFF) demonstrated in Figure 1. The parameters are defined according to [30] as shown in Table 1. The element of motion in BFF is given in the following vectors.

$$
\begin{gathered}
v=\left[\begin{array}{ll}
v_{1} & v_{2}
\end{array}\right]^{T} \\
v_{1}=\left[\begin{array}{lll}
u & v & w
\end{array}\right]^{T} \text { Linear velocities } \\
v_{2}=\left[\begin{array}{lll}
p & q & r
\end{array}\right]^{T} \text { Angular velocities }
\end{gathered}
$$

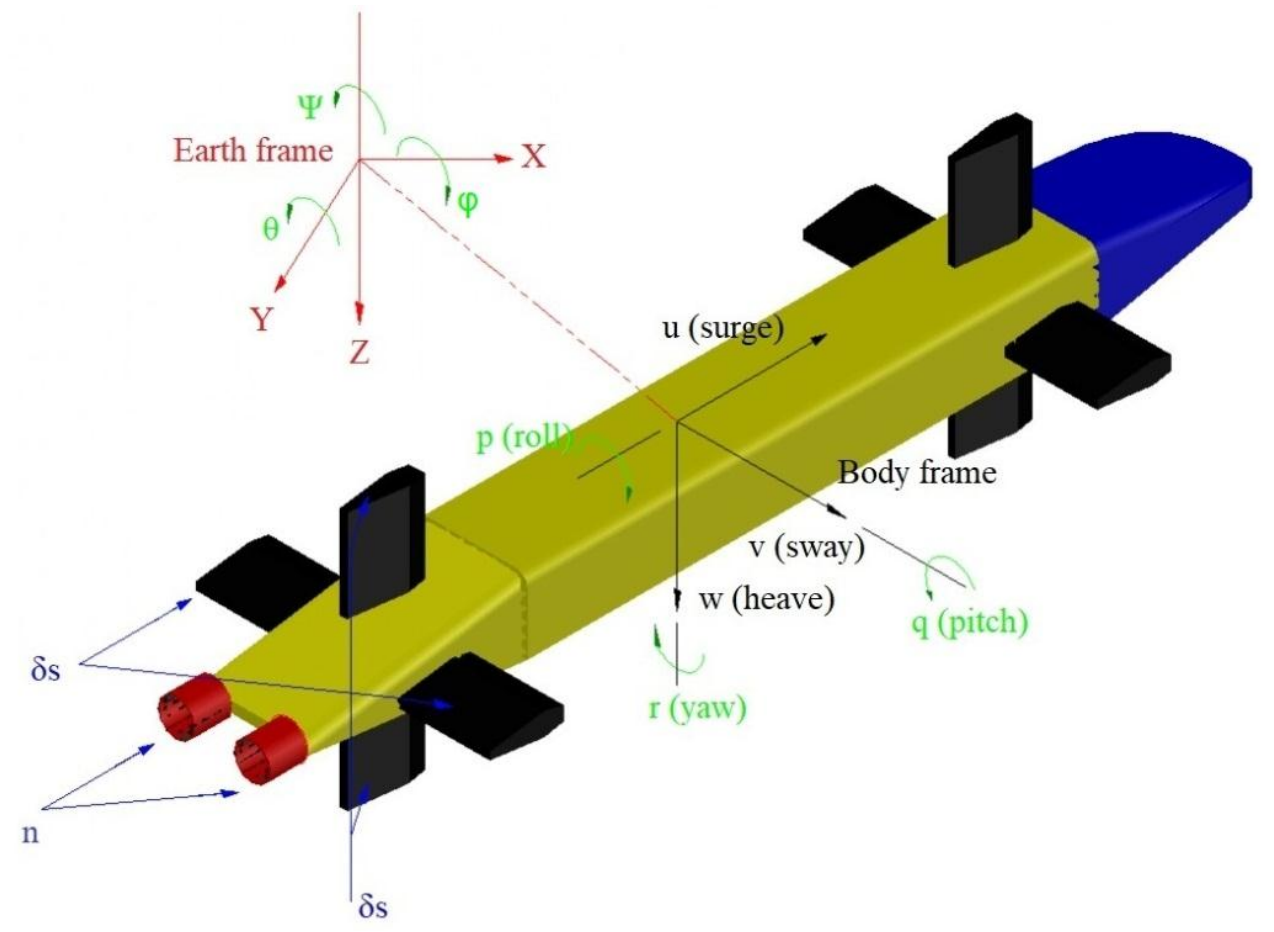

Figure 1. The schematic of NSP II BFF and EFF [29] 
Table 1. The Notations for marine vessel [30]

\begin{tabular}{|c|c|c|c|}
\hline $\begin{array}{c}\text { Degree of } \\
\text { Freedom }\end{array}$ & $\begin{array}{c}\text { Forces \& } \\
\text { Moments }\end{array}$ & $\begin{array}{c}\text { Linear and } \\
\text { Angular } \\
\text { Velocities }\end{array}$ & $\begin{array}{c}\text { Position and } \\
\text { Euler Angles }\end{array}$ \\
\hline Surge & $X$ & $u$ & $x$ \\
\hline Sway & $Y$ & $v$ & $y$ \\
\hline Heave & $Z$ & $p$ & $\phi$ \\
\hline Roll & $M$ & $q$ & $\theta$ \\
\hline Pitch & $N$ & $r$ & $\psi$ \\
\hline Yaw & & & \\
\hline
\end{tabular}

The position and attitude vectors of the BFF with referring to EFF express as:

$$
\begin{aligned}
& n=\left[n_{1} n_{2}\right]^{T}
\end{aligned}
$$

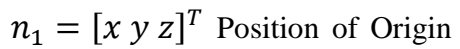

$$
\begin{aligned}
& n_{2}=\left[\begin{array}{lll}
\phi & \theta & \psi
\end{array}\right]^{T} \text { Angles orientation of roll }(\phi) \text {, }
\end{aligned}
$$

pitch $(\theta)$ and yaw $(\psi)$

The nonlinear dynamic equation of motion in the BFF is defined as follows

$$
\mathrm{M} \dot{v}+\mathrm{C}(\mathrm{v}) \mathrm{v}+\mathrm{D}(\mathrm{v}) \mathrm{v}+\mathrm{G}(\mathrm{\eta})=\tau
$$

Where, $M \in \Re^{6 \times 6}$ is in inertia matrix, $C(v) \in \Re^{6 \times 6}$ is Coriolis and centripetal matrix, $D(v) \in \Re^{6 \times 6}$ is damping matrix, $G(\eta) \in \Re^{6 \times 1}$ is vector of buoyancy/gravitational forces/moments matrix and $\tau \in \Re^{6 \times 1}$ is vector of control inputs relating to forces and moments acting on vehicle.

The control input vector $\tau$ has three components as:

$$
\tau=\left[\delta_{r}, \delta_{e}, n\right]
$$

Where $\delta_{e}$ is elevator deflection, $\delta_{r}$ is rudder deflection and $n$ is propeller revolutions.

\subsection{Linearized equation of motion}

The kinematic equation of motion in depth plane is given by:

$$
\begin{gathered}
\dot{z}=-u(\sin \theta)+w(\cos \theta) \\
\dot{\theta}=q
\end{gathered}
$$

$u$ and $w$ representing surge and heave velocities of the vehicle. In steady state motion of a vehicle, $\theta_{0}=q_{0}=$ $\phi_{0}=0$. Hence kinematic equation is rewritten as:

$$
\begin{aligned}
\dot{z} & =-\theta u_{0}+w \\
\dot{\theta} & =q
\end{aligned}
$$

The depth plane dynamics equation of motion is obtained by setting all state vector related to steering plane to zero ( $v=p=r=\phi=\psi=0$ ). Hence, equation of motion in depth plane express as:

$$
\begin{array}{r}
m\left(\dot{w}-u_{0} q\right)=Z \\
I_{y} \dot{q}=M
\end{array}
$$

The external forces $X, Z$ and moment $M$ described by hydrodynamic added mass, linear damping and the effects of the elevator deflection, depth plane model is simplified as:

$$
\begin{gathered}
Z=Z_{\dot{w}} \dot{w}+Z_{\dot{q}} \dot{q}+Z_{w} w+Z_{q} q+Z_{\delta} \delta_{s} \\
M=M_{\dot{w}} \dot{W}+M_{\dot{q}} \dot{q}+M_{w} w+M_{q} q-m g\left(z_{G}-\right. \\
\left.z_{B}\right) \sin \theta+M_{\delta} \delta_{s}
\end{gathered}
$$

From (10), (11) and (12), linearized model in vertical plane equation of motion can be written in the following form.

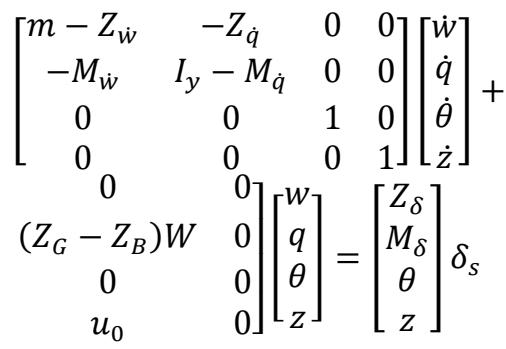

As in [31], the heave velocity during diving is small (less than $0.05 \mathrm{~m} / \mathrm{s}$ ), thus term containing $w$ and $\dot{w}$ can be neglected. Since $w$ and $\dot{w}$ are neglected, the mathematical model of vertical plane as in (13) can be expressed in state space form as:

$$
\left[\begin{array}{c}
\dot{q} \\
\dot{\theta} \\
\dot{Z}
\end{array}\right]=\left[\begin{array}{ccc}
\frac{M_{q}}{I_{y}-M_{\dot{q}}} & \frac{-W\left(Z_{G}-Z_{B}\right)}{I_{y}-M_{\dot{q}}} & 0 \\
1 & 0 & 0 \\
0 & -u_{0} & 0
\end{array}\right]\left[\begin{array}{c}
q \\
\theta \\
z
\end{array}\right]+\left[\begin{array}{c}
\frac{M_{\delta}}{I_{y}-M_{\dot{q}}} \\
0 \\
0
\end{array}\right] \delta_{s}
$$

Where $M_{q}$ is pitch moment due to $q, M_{\dot{q}}$ is pitch moment due to time rate of change of $q, I_{y}$ is vehicle inertia around the pitch axes, $W$ is AUV weight, $Z_{G}$ is center of gravity, $Z_{B}$ is center of buoyancy, $u_{0}$ is designed velocity and $M_{\delta}$ is fin lift coefficient. Then, (14) can be expressed as:

$$
\dot{x}(t)=A x(t)+B u(t) y(t)=C x(t)
$$

\section{Depth Control of AUV NPS II in DSMC}

DSMC is designed in this section to control desired depth of NSP II AUV. Considering discrete-time linear system described by

$$
\begin{gathered}
(k+1)=(k)+\Gamma u(k) \\
(k+1)=(k)
\end{gathered}
$$

Where $x(k) \in \Re^{n}$ is the state vector, $u(k) \in \Re^{m}$ is the control input, $\Phi \in \Re^{n \times n}$ and $\Gamma \in \Re^{n \times n}$ are the system matrices.

The sliding surface in discrete time is given by

$$
S(k)=C_{s} e(k)=C_{s}\left(x_{r}(k)-x(k)\right)
$$

Where $e(k)$ is the tracking error, $x_{r}(k)$ is reference input and $C_{s} \in \Re^{m}$ is the sliding matrix selected such that $C_{S} \Gamma$ is nonsingular. 
The system is designed to steer the state trajectory to the origin when travelling along switching surface. Without loss of generality, the following linear sliding surface is defined as:

$$
S(k)=0
$$

Considering (18), the first-time derivative of the sliding surface is given by:

$$
\begin{aligned}
S(k+1)-S(k)= & C_{S}\left[x_{r}(k+1)-x(k+1)\right] \\
& -C_{S}\left[x_{r}(k)-x(k)\right] \quad(20)
\end{aligned}
$$

Substituting (16) into (20), the first derivative of sliding surface is rewritten as:

$$
\begin{array}{r}
S(k+1)-S(k)=C_{s}\left[x_{r}(k+1)-\Phi x(k)-\Gamma u(k)\right] \\
-C_{s}\left[x_{r}(k)-x(k)\right]
\end{array}
$$

A discrete-time extension of reaching law approach was proposed by Gao [32].The reaching law in this case is given by

$$
S(k+1)-S(k)=-q T S(k)-\varepsilon \operatorname{Tsgn}(S(k))
$$

Where $\varepsilon>0, q>0$ and $1-q T>0$ and $T$ is the sampling time.

Hence, the control law is expressed as:

$$
\begin{array}{r}
u(k)=-\left(C_{s} \Gamma\right)^{-1}\left[-C_{s} x_{r}(k+1)+C_{s} \Phi x(k)+C_{s} x_{r}(k)\right. \\
\left.-C_{s} x(k)-q T s(k)-\varepsilon T s g n(s(k))\right]
\end{array}
$$

Following steps are to obtain sliding gain matrix $C_{s}$.

Firstly, by substitute (22) into (23), it yields

$$
\begin{aligned}
& X(k+1)=(\Phi-\Gamma K) X(k) \\
& \text { Where } K=\left(C_{S} \Gamma\right)^{-1} C_{S} \Phi
\end{aligned}
$$

Hence, the sliding gain matrix $C_{s}$ becomes the solution of the following equation.

$$
\begin{gathered}
C_{s}(\Phi-\Gamma K)=0 \\
C_{S} \Gamma=I
\end{gathered}
$$

Where $I$ is an identity matrix and (27) is to ensure that $C_{S} \Gamma$ is full rank. Using (26), (27) can be replaced by $C_{S} \Phi=K$ and thus above equations can be rewritten as:

$$
C_{s}[\Phi-\Gamma]=[K I]
$$

Finally, the sliding matric $C_{S}$ is given by

$$
C_{s}=[K I][\Phi \Gamma]^{+}
$$

Where + is representation of matrix pseudo-inverse. The feedback matrix $K$ can be obtained by adopting (27) into LQR controller [1].

\section{Simulation on Depth Control}

To demonstrate effectiveness of discrete sliding mode controller, simulation has been made on AUV NSP II specifically on depth control system. A depth control is simulated with the full linear six degree of freedom (DOF) and simplified equation of motion reduced to heave $z$ and pitch $\theta$. The AUV NSP II is $5.3 \mathrm{~m}$ in length. Its weight in air is $5443.4 \mathrm{~kg}$ and has natural buoyancy in water. The flooded mass in water is about $53400 \mathrm{~N}$. The maximum speed in calm water is $3 \mathrm{~m} / \mathrm{s}$ [33].Added mass inertia, $M_{\dot{q}}$ is $-1.7 \times 10^{-2}$ and moment of inertia in $y$-axes, $I_{y}$ is $13587 \mathrm{nms}^{2}$.

To illustrate an effectiveness of DSMC, discrete PID controller is used as a comparative analysis. Step response simulations are performed in heave and pitch DOF at $T=0.2$. PID controller is widely used due to its reliability and simplicity but it's difficult to tune the parameters in discrete PID controllers to achieve optimal performances. PID gain setting as in Table 2 .

Table2. PID Gain value

\begin{tabular}{|c|c|}
\hline Gain & Value \\
\hline$K p$ & -0.1629 \\
\hline$K d$ & $-4.0117 \mathrm{e}-05$ \\
\hline$K i$ & 3.09681 \\
\hline
\end{tabular}

Using (29), the sliding matrix $C_{s}$ is given by

$$
C_{s}=[1.6136-1.9098-0.225]
$$

The reaching law parameters are selected as follow

$$
q=0.4, \varepsilon=0.01
$$

Dynamic model of AUV NSP II for diving control was carried out by setting up the desired depth is $8 \mathrm{~m}$. Fig.2 shows result of diving control responded under ideal conditions. The settling time taken is 180 s and 250s for DSMC and discrete PID respectively. DSMC proved shortest settling time due to its sensitivity to parameter variations. Fig. 3 indicates elevator deflection commanded by the controller to AUV as an input and AUV pitch angle response as output shown in Fig.4. The result illustrated elevator deflection response by DSMC is smoother and sensible. In contrast to discrete PID controller, elevator response shows lumpy motion to pitch angle of AUV. From this simulation, it's clearly demonstrated that the proposed method improved controller performances, strong robustness and satisfactory stability. 


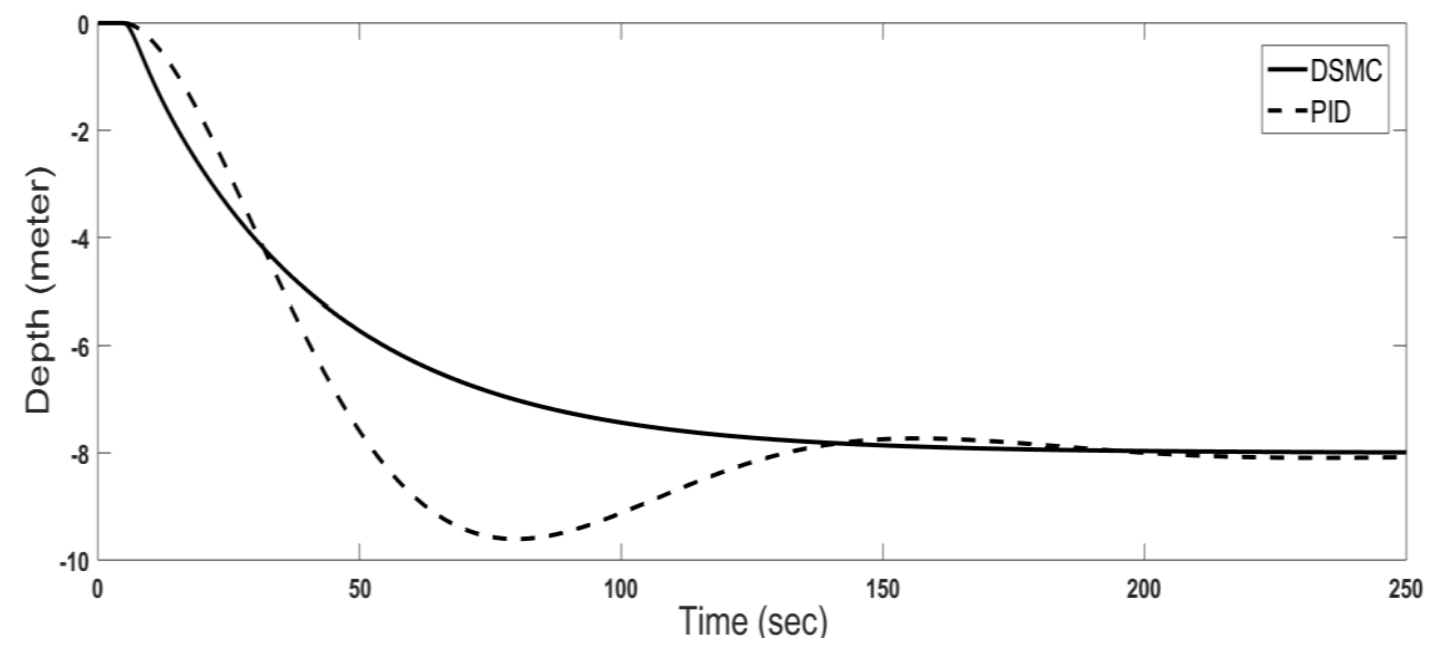

Figure 2. Vehicle depth response

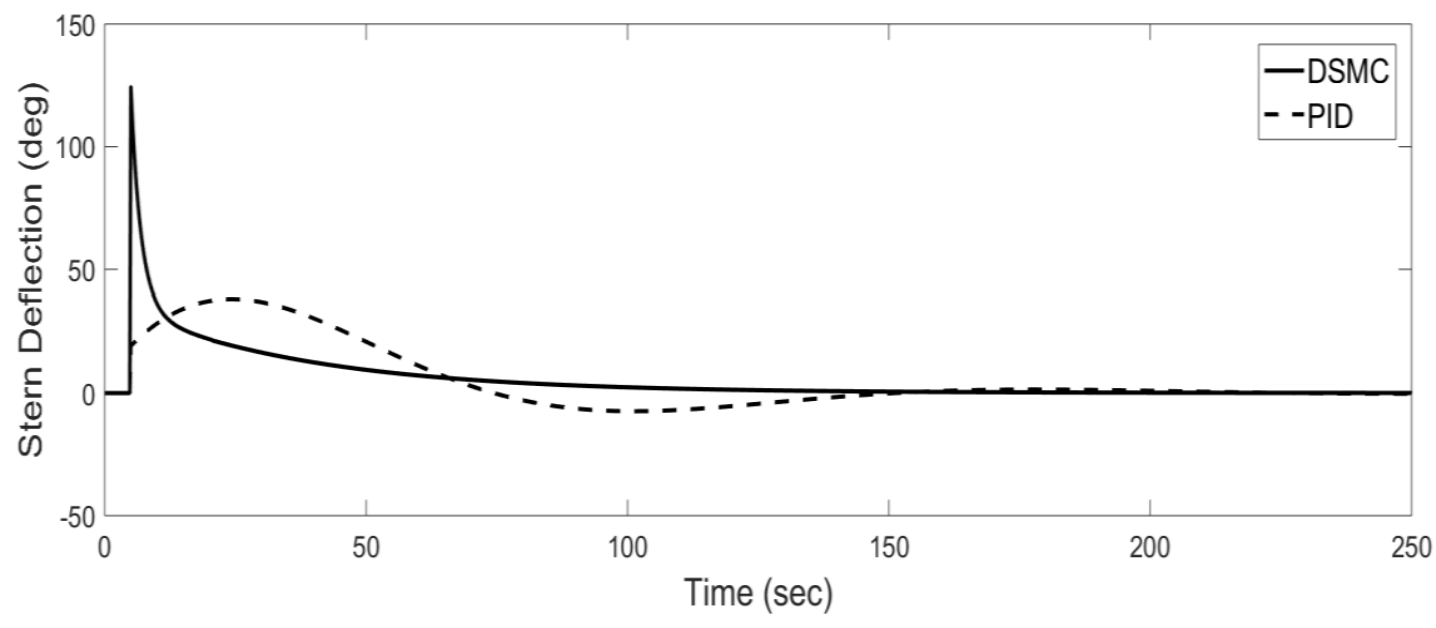

Figure 3. Vehicle elevator deflection response

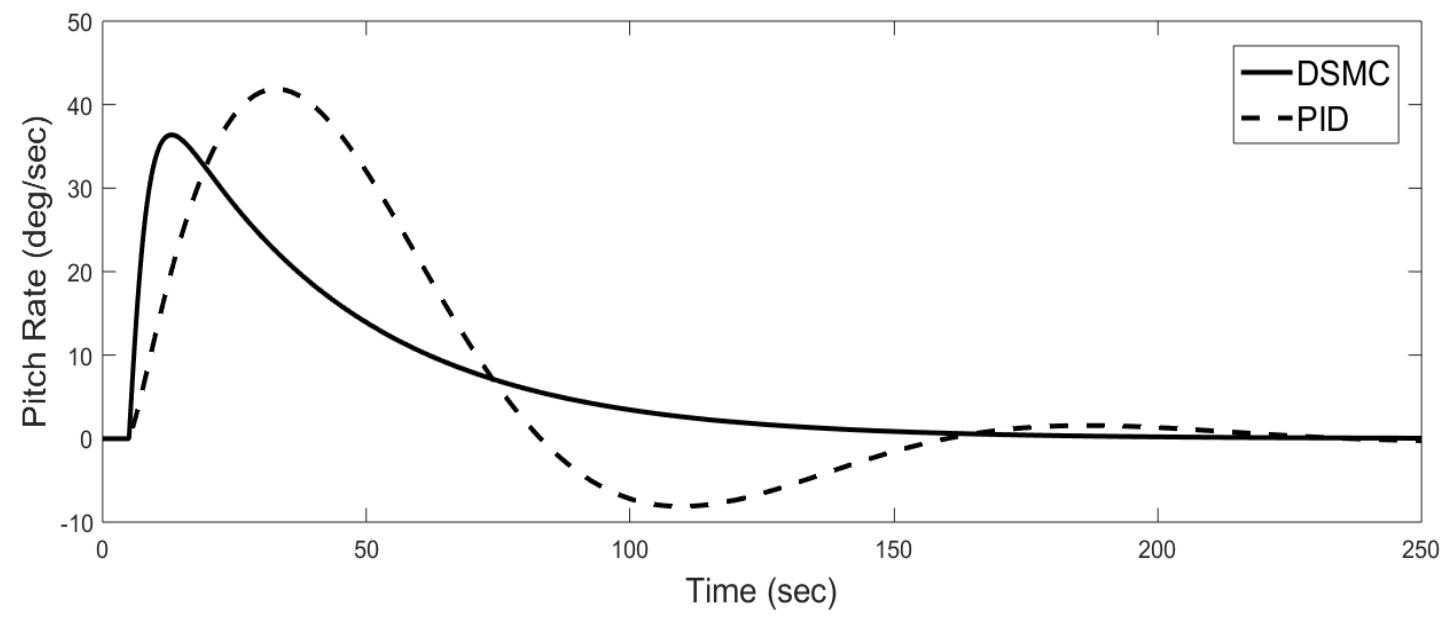

Figure 4. Vehicle pitch rate response 


\section{Conclusions}

In this study, the algorithm of discrete SMC was proposed. The reason of choosing is due to robustness against external disturbance and uncertain dynamics. Finally, the simulation and experiment result show excellent performance in depth control of NSP AUV II. Two control methods for depth regulation consisting of discrete SMC and discrete PID and each performance was compared via simulation. The result indicates discrete-time SMC has higher control precision, faster convergence and stronger robustness than discrete-time PID. In the future work, disturbance will be added into algorithm and both controller performances will be tested via simulation and experiment.

\section{Acknowledgements}

The authors would like to thank Universiti Tun Hussein Onn Malaysia (UTHM) for TIER 1 grant Vot H148, GPPS grant Vot H316 and AdMiRe FKEE for the support.

\section{REFERENCES}

[1] B. Draženović, Č. Milosavljević, and B. Veselić, Comprehensive approach to sliding mode design and analysis in linear systems, no. January. Springer, 2013.

[2] M. Nahon, "A simplified dynamics model for autonomous underwater vehicles," in Proceedings of Symposium on Autonomous Underwater Vehicle Technology, 2002, pp. 373-379.

[3] K. Isa, M. R. Arshad, and S. Ishak, "A hybrid-driven underwater glider model, hydrodynamics estimation, and an analysis of the motion control," Ocean Eng., vol. 81, pp. 111-129, 2014.

[4] Donald P. Brutzman, "A Virtual World For An Autonomous Underwater Vehicle," (Phd Thesis), Naval Postrgaduate School, Monterey, California, 1994.

[5] A. Healey and D. Marco, "Slow speed flight control of autonomous underwater vehicles: Experimental results with NPS AUV II," in The Second International Offshore and Polar Engineering Conference, 1992.

[6] J. Guerrero, E. Antonio, A. Manzanilla, J. Torres, and R. Lozano, "Autonomous Underwater Vehicle Robust Path Tracking: Auto-Adjustable Gain High Order Sliding Mode Controller," IFAC-Papers Online, vol. 51, no. 13, pp. 161166, 2018.

[7] M. Farhan, "Sliding Mode Control of Autonomous Under Water Vehicle" (Master Thesis), Muhammad Farhan Faculty of Engineering, Capital University of Science \& Technology" Thesis, May, 2017.

[8] Y. S. Song and M. R. Arshad, "Sliding mode depth control of a hovering autonomous underwater vehicle," in Proceedings
- 5th IEEE International Conference on Control System, Computing and Engineering, ICCSCE 2015.

[9] B. Ullah, M. Ovinis, M. B. Baharom, M. Y. Javaid, and S. S Izhar, "Underwater gliders control strategies: A review," in 2015 10th Asian Control Conference: Emerging Control Techniques for a Sustainable World, ASCC 2015.

[10] L. Qiao and W. Zhang, "Adaptive Second-Order Fast Nonsingular Terminal Sliding Mode Tracking Control for Fully Actuated Autonomous Underwater Vehicles," IEEE J. Ocean. Eng., vol. 44, no. 2, pp. 363-385, 2019.

[11] R. Cui, X. Zhang, and D. Cui, "Adaptive sliding-mode attitude control for autonomous underwater vehicles with input nonlinearities," Ocean Eng., vol. 123, pp. 45-54, 2016.

[12] Z. Chu, X. Xiang, D. Zhu, C. Luo, and D. Xie, “Adaptive Fuzzy Sliding Mode Diving Control for Autonomous Underwater Vehicle with Input Constraint,” Int. J. Fuzzy Syst., no. November, pp. 10-11, 2017.

[13] B. Wang, "On Discretization of Sliding Mode Control Systems On Discretization of Sliding Mode Control Systems (Phd Thesis)," RMIT University, 2008.

[14] D. P. Singh, S. Agarwal, and U. K. Gupta, "A Technical Review on Discrete-Time Sliding Mode Controller for Linear Time-Varying Systems,” Int. J. Eng. Tech. Res., no. 5, pp. 289-291, 2014.

[15] Y. Feng, C. Xue, X. Yu, and F. Han, "On A Discrete-Time Quasi-Sliding Mode Control," in Proceedings of IEEE International Workshop on Variable Structure Systems, vol. 2, pp. 251-254, 2018.

[16] P. Lee, S. Hong, and Y. Lim, "Self-tuning control of autonomous underwater vehicles based on discrete variable structure system," Ocean. '97. MTS/IEEE Conf. Proc., vol. 2, pp. 902-909, 1997.

[17] P. M. Lee, S. W. Hong, Y. K. Lim, C. M. Lee, B. H. Jeon, and J. W. Park, "Discrete-time quasi-sliding mode control of an autonomous underwater vehicle," IEEE J. Ocean. Eng., 1999.

[18] S. Zhang, J. Yu, and A. Zhang, "Discrete-time quasi-sliding mode control of underwater vehicles," Proc. World Congr. Intell. Control Autom., pp. 6686-6690, 2010.

[19] B. Wu, S. Li, and X. Wang, "Discrete-time adaptive sliding mode control of autonomous underwater vehicle in the dive plane," in Springer-Verlag Berlin Heidelberg, , vol. 5928 LNAI, pp. 157-164, 2009.

[20] T. M. and S. M. Y. Dote, "Microprocessor-Based Force Control for Manipulator Using Variable Structure with Sliding Mode," IFAC Proc. Vol., vol. 16, no. 16, pp. 145149,1983

[21] Y. I. and O. K. Sami Z. Sarpturk, "On the Stability of Discrete-Time Sliding Mode Control System," IEE Conf. Publ., vol. 1, no. 332, pp. 930-932, 1987.

[22] K. Furuta, "Sliding mode control of a discrete system," Syst. Control Lett., vol. 14, no. 2, pp. 145-152, Feb. 1990.

[23] W. Gao and J. C. Hung, "Variable Structure Control of Nonlinear Systems: A New Approach," IEEE Trans. Ind. Electron., 1993. 
[24] A. Bartoszewicz, Discrete-time quasi-sliding-mode control strategies, IEEE Transections on Automation on Industrial Electronics, vol. 45. 1998.

[25] I. Bsili, J. Ghabi, and H. Messaoud, "Discrete sliding mode control of inverted pendulum," in World Symposium on Mechatronics Engineering and Applied Physics, 2015, no. November 2018.

[26] M. S. G. Dias, "Discrete time sliding mode control strategies applied to a multiphase brushless DC machine" Kassel University press GmbH,Kassel (Phd Thesis),2017.

[27] D. H. Shah, "Discrete-Time Sliding Mode Control with Disturbance Estimator for Networked Control System," (Phd Thesis), Gujarat Technological University, Ahmedabad, 2018 .

[28] W. et al Gao, "Discrete-Time Variable Structure Control Systems," IEEE Trans. Ind. Electron., vol. 42, no. 2, pp. $117-122,1995$.

[29] T. I. Fossen, Guidance and control of ocean vehicles, 4th ed. Wiley New York, 1994.

[30] E Snam, "Nomenclature for treating the motion of a submerged body through a fluid JR," New York Tech. Res. Bull., p. 1, 1950.

[31] B. Jalving, “The NDRE-AUV Flight Control System,” IEEE Journal of Oceanic Engineering, vol. 19, no. 4. pp. 497-501, 1994.

[32] W. Gao, Y. Wang, and A. Homaifa, "Discrete-Time Variable Structure Control Systems," IEEE Trans. Ind. Electron., 1995.

[33] F. Gerdönmez, "Simulation of Motion of an Underwater Vehicle," (Phd Thesis), Middle East Technical University, 2007. 